BONPLANDIA 20(2). 2011

ISSN: 0524-0476

\title{
LA "YERBA MATE" (ILEX PARAGUARIENSIS, AQUIFOLIACEAE) EN TEMPRANOS ESCRITOS RIOPLATENSES DE BONPLAND Y SU REAL DISTRIBUCIÓN GEOGRÁFICA EN SUDAMÉRICA AUSTRAL
}

\author{
GUSTAVO C. GIBERTI
}

\begin{abstract}
Summary: Giberti, G. C. 2011. "Yerba mate" (Ilex paraguariensis, Aquifoliaceae) according early Río de La Plata Bonpland's handwritings: its southern South American geographical distribution. Bonplandia 20(2): 203-212.
\end{abstract}

\begin{abstract}
A transcription and a critical review on Aimé Bonpland's scarcely known manuscripts dealing with the geographical distribution of wild "yerba mate" (Ilex paraguariensis A. St. Hil., Aquifoliaceae) trees in South America is presented here. Among other data, Bonpland's assumptions on the occurrence of wild "yerba mate" individuals within present day Uruguayan boundaries are matched with modern voucher specimens of this species collected in Uruguay, and also with miscellaneous bibliographical sources, including details from later reports from Bonpland himself, and also from other contemporary naturalists who travelled through this country in the 1820's and 1830's, as well as with phytogeographical information. As a conclusion, it is supposed that these Bonpland's notes, written during his early years of residence at the Río de La Plata basin, contain mistaken information that the same botanist did not report again, as his knowledge of the Argentinian Delta, the Uruguayan environment and the "yerba mate" got deeper.
\end{abstract}

Key words: Aimé Bonpland, Ilex paraguariensis, manuscripts, Delta, Uruguay, chorology, maté.

Resumen: Giberti, G. C. 2011. La "yerba mate" (Ilex paraguariensis, Aquifoliaceae) en tempranos escritos rioplatenses de Bonpland y su real distribución geográfica en Sudamérica austral. Bonplandia 20(2): 203-212.

Se presentan la descripción y el comentario crítico de manuscritos poco conocidos de Amado Bonpland que conciernen a la distribución geográfica del árbol que produce la "yerba mate" (Ilex paraguariensis, Aquifoliaceae) en Sudamérica. Entre otros datos, las afirmaciones de Bonpland sobre la existencia de individuos silvestres de "yerba mate" creciendo dentro de las fronteras actuales de la República Oriental del Uruguay son confrontadas con ejemplares de herbario recientemente coleccionados en Uruguay y con diversas fuentes bibliográficas, incluyendo datos adicionales del mismo Bonpland y de otros naturalistas contemporáneos del botánico francés que viajaron por ese país en las décadas de 1820 y 1830, a la par de revisar informaciones fitogeográficas existentes. Se concluye que estos escritos de Bonpland, redactados durante sus primeros años de residencia en la Cuenca del Plata, poseen informaciones incorrectas que no se conoce que el mismo naturalista haya vuelto a reportar, en la medida en que sus conocimientos sobre el Delta argentino, el ambiente uruguayo y sobre la "yerba mate" se fueron incrementando.

Palabras clave: Amado Bonpland, manuscritos, Ilex paraguariensis, corología, Delta, Uruguay, "yerba mate".

${ }^{1}$ IQUIMEFA (CONICET, FFyB), Junín 956, 1113-Buenos Aires, Argentina. e-mail: giberti@sinectis.com.ar 


\section{Introducción}

El botánico francés Aimé Bonpland (17731858), antiguo compañero de viajes del barón Alexander von Humboldt (1769-1859) en su famosa exploración de las tierras equinocciales de América (1799-1804), que fuera posteriormente Superintendente de los jardines de la Emperatriz Josephine en las residencias Malmaison y Navarre (1808-1814), permaneció casi la mitad de su vida (desde 1817 hasta su muerte en 1858) residiendo en varios países de la cuenca del Plata. Asimismo, Bonpland viajó reiteradamente a través de esta extensa región de Sudamérica (Hamy, 1906; Castellanos, 1963; Hossard, 2001; Bell, 2010). Allí, Bonpland no solamente se dedicó a satisfacer su interés permanente en las Ciencias Naturales, sino que ejerció la medicina, fue agricultor y ganadero, a la par de ser testigo de complicados eventos políticos regionales, en algunos de los cuales se involucró personalmente hasta el punto de sufrir las consecuencias de varios conflictos armados en un período de las décadas de 1830 a 1850 (Krapovickas, 2008). Como un resultado indeseable de sus múltiples y variadas actividades en esa etapa de su vida, sus publicaciones científicas prácticamente desaparecieron en esos años (Castellanos, 1963; Lourteig, 1977).

No obstante, gran cantidad de sus manuscritos de esa época han sobrevivido, atestiguando su permanente interés en el conocimiento científico de su ambiente, y todavía merecen estudio. De esos escritos, algunos de los relacionados con la "yerba mate", también conocida como "té del Paraguay" o "maté" (Ilex paraguariensis A. St. Hil., Aquifoliaceae) se considerarán aquí.

\section{Bonpland y las colecciones de Ilex paraguariensis, la sistemática del género y el cultivo de "yerba mate"}

Uno de los mayores temas de interés que tuvo para Bonpland la región rioplatense fue el conocimiento científico $y$, por motivos económicos, inclusive la producción misma de la "yerba mate". A pesar de haber sido el primer botánico europeo post-linneano en proponer un binomial y redactar la descripción botánica del árbol productor de la "yerba mate" (Bonpland denominó Ilex theaezans a esta especie tempranamente -circa 18181820-), posteriormente ya advirtió que el nombre de su autoría no fue aplicado a la misma entidad: en efecto, sus estudios, que permanecieron varios años inéditos, fueron conocidos por la comunidad científica varias décadas después de la publicación de la primera descripción válida y efectiva del vegetal por Auguste de Saint Hilaire, quien lo llamó Ilex paraguariensis en 1823 (Saint Hilaire 1823 "1822"; Giberti, 1990).

Asimismo, otras contribuciones botánicas de Bonpland a la taxonomía de algunos parientes co-genéricos de la yerba mate genuina -a los que describió y denominó Ilex brevifolia, $I$. crepitans, I. gigantea, I. humboldtiana e I. ovalifolia-, a pesar de haber sido descriptos en su cuaderno botánico Journal de Botanique (Domínguez, 1924), permanecieron como nombres inéditos hasta ser publicados en un período de tiempo que transcurre entre 1861 y un cuarto de siglo después de la muerte del botánico francés. Así, sus descripciones botánicas y nombres fueron publicados o considerados sinónimos de otras especies de Ilex L. por John Miers (1861): I. gigantea, I. humboldtiana, I. ovalifolia, I. crepitans, y por Johann Münter (1883): Ilex brevifolia.

Hacia fines del siglo XIX y principios del siguiente (Brown, 1892; Loesener, 1901), ya todos esos nombres eran considerados sinónimos de otras especies de Ilex (Giberti, 1988). Sin embargo, la influencia de Bonpland sobre el cultivo y las modalidades de producción de la yerba mate se hizo sentir, incluso desde los últimos años de su vida (Quesada, 1857). Asimismo, sus enseñanzas sobre una forma más adecuada de explotar los yerbales naturales y sus intentos de dominar el cultivo de este árbol sugiriendo mejoras en las técnicas destinadas a posibilitar la germinación de las semillas, influyeron en las modalidades de producción de yerba mate hasta las primeras décadas del siglo XX.

\section{Los manuscritos de Bonpland y la distribución de Ilex paraguariensis}

En forma análoga, parte de los escritos más 
conocidos de Bonpland sobre la distribución geográfica de los árboles silvestres de "yerba mate" se empezaron a publicar durante sus últimos años de vida (Delessert, 1854; Neumann, 1858; Quesada, 1857). Pero es importante recordar que el concepto de Bonpland sobre "yerba mate" no solamente involucraba la especie que hoy en día se reconoce como productora de yerba mate auténtica -Ilex paraguariensis-, sino que asimismo abarcaba otras especies del mismo género. Eventualmente, algunos de estos datos, si bien algo confusos en términos estrictamente sistemáticos, volvieron a publicarse luego de la muerte de Bonpland (Bonpland, 1867, 1949). En consecuencia, ciertas informaciones distorsionan el panorama de la corología de esta especie en América del Sur.

Entre estos asuntos, en ocasión del reciente sesquicentenario de la desaparición de Bonpland (2008), he revisado una serie de manuscritos del botánico francés que se conservan en el Archivo Bonpland de la Facultad de Farmacia y Bioquímica dependiente de la Universidad de Buenos Aires. Entre ellos, llamó mi atención un antiguo cuaderno de notas, desafortunadamente bastante deteriorado -el manuscrito $\mathrm{N}^{\mathrm{o}} 2044$ , escrito supuestamente entre 1818 y 1824 , que consiste de varios asuntos bien diferentes que fueron redactados por Bonpland. Se trata de una serie de diversas anotaciones, algunas notoriamente inconclusas, tales como observaciones termométricas registradas por Aimé en Buenos Aires entre enero y octubre de 1820, datos similares anotados durante su viaje a Corrientes (1820-1821), un detallado relato de su navegación por el rio Paraná Guazú y cursos de agua aledaños en agosto - septiembre de 1819, notas sobre el idioma de los indios paraguayos, apuntes sobre la fabricación de licores durante su cautiverio en Paraguay, etc. Asimismo, las notas sobre la "yerba mate" (Fig. 1) que motivan este trabajo también se encuentran en el mismo cuaderno manuscrito.

Entre estos manuscritos presuntamente tan tempranos de la etapa "rioplatense" de su vida (Bonpland había arribado al puerto de Buenos Aires pocos años antes), el naturalista daba cuenta del hallazgo del árbol de la "yerba mate" (aquí indudablemente se trata de Ilex paraguariensis) en la isla Martín García en 1818. Además, en ellos el botánico consideraba una serie de datos adicionales que trataré en el presente trabajo, a pesar que parte de ellos ya hayan sido someramente comentados (Galarza, 1914; Muello, 1946; Martínez Crovetto, 1995).

Sigue entonces a este párrafo la transcripción del fragmento de esos escritos (Bonpland, 1818-1824a), probablemente redactados entre fines de 1818 y agosto de 1819 (Fig. 1) que interesa comentar aquí:

"Hierba del Paraguay o Mate"

"En Décembre $1818 \mathrm{j}$ ' ai trouvé l' arbre du maté dans 1' île de Martín García; de retour à B. Ayres j'ai préparè les feuilles et elles m'ont produit un maté qui ne differait en rien du meilleur mate. Le Docteur Pueyrredón m 'avait assuré qu'il avait vu 1' arbre du maté à Martín García et personne ne la connaissait dans cette île lorsque; $\mathrm{j}$ 'suis allé; plusieurs paraguayos même qui ètaient là, qui avait travaillé comme journalier, dans les yerbales ne le reconnarait pas".

"Plusieur personnes assurent avoir vu la même plante dans les îles du Paraná nommé Las Palomas, Los Platos y Botijas on m'a aussi assuré qui elle existait en el arroyo de las Piedras et c 'est du cet arroyo où Mr. Dapin on a aporté deux petits pièces à Mr. le Doct. Pueyrredón qui les culture à Sn. Isidro depuis 1818. Quelques personnes pretendent aussi que cettes plantes se trouve dans 1 ' îles de Pay Caraby qui selon plusieurs a èté cultiveè par les jesuites. $\mathrm{D}$ 'aprés de tels renseignements on doit visiter avec soin les diverses îles du Paraná.

On m `a aussi assuré qu 'il y avait beaucoup de pièces du maté dans les îles de 1 ' Uruguay, celles du Rio Negro et surtout a la Bande Orientale. Plusieur personnes dirent avoir fait de 1 'herbe au Rincón de Aedo ou de las Gallinas. Dn. Bruno Reynal a vu cette plante près de las Maulas (rivières) dans un endroit appelé los Cerritos dit à trois lieues de distance de la village de Soriano. Dans la Sierra de Santa María qui se trouve à quelque distance de 1 ' Herbidero on dit que 1 'arbre du mate y est très abondant."

Es interesante mencionar que a pesar de la información de Bonpland sobre la existencia de Ilex paraguariensis en el Delta de los ríos 


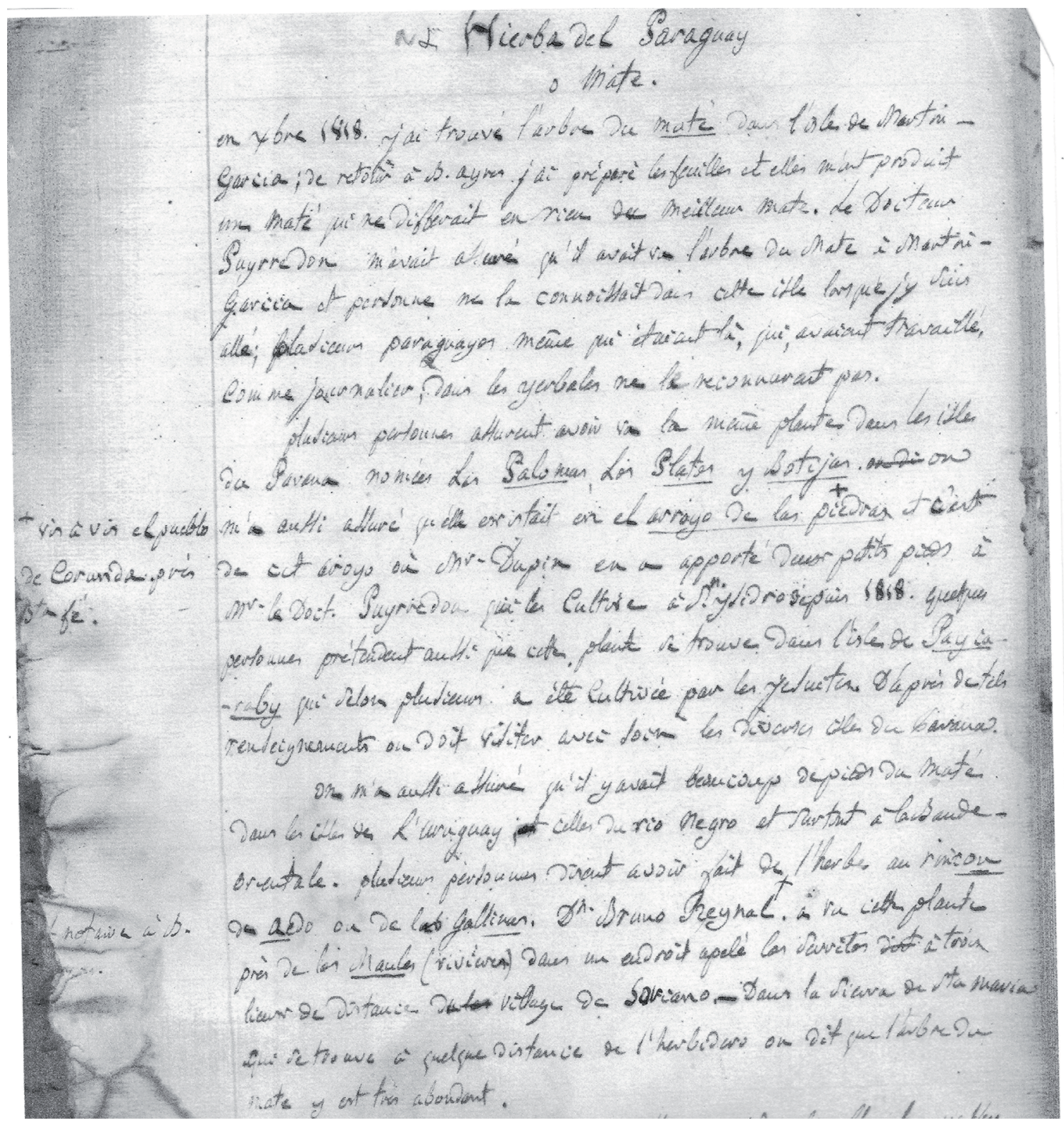

Fig. 1. Fragmento de la página perteneciente al documento $\mathrm{N}^{\circ} 2044$ del Archivo Bonpland del Museo de Farmacobotánica "Juan A. Domínguez" donde el naturalista trata la "yerba mate" (Ilex paraguariensis) y su existencia en Uruguay y en el Delta de los ríos Paraná y Uruguay (Argentina).

Paraná y Uruguay (Fig. 2), el naturalista después la buscó infructuosamente en la zona durante su viaje por el Paraná Guazú en 1819 (Bonpland, 1818-1824b; Bell, 2010). Además, el autor del presente trabajo jamás ha encontrado ni un solo ejemplar de herbario de esta especie proveniente de la zona, a pesar de la existencia de otras referencias bibliográficas (Spegazzini, 1905). Análogamente, la presunta presencia de este árbol creciendo en condiciones silvestres nunca se confirmó ni para las provincias de Buenos Aires (e.g. en las islas Botijas y Paycarabí), ni Entre Rios -e.g. arroyo Las Piedras, isla Las Palomas- (Burkart \& Bacigalupo, 2005; Cabrera, 1965; Giberti, 1979, 1994). Sobre la supuesta existencia de I. paraguariensis en las islas Paycarabí, y su posible cultivo allí por los misioneros jesuitas, presumo que 


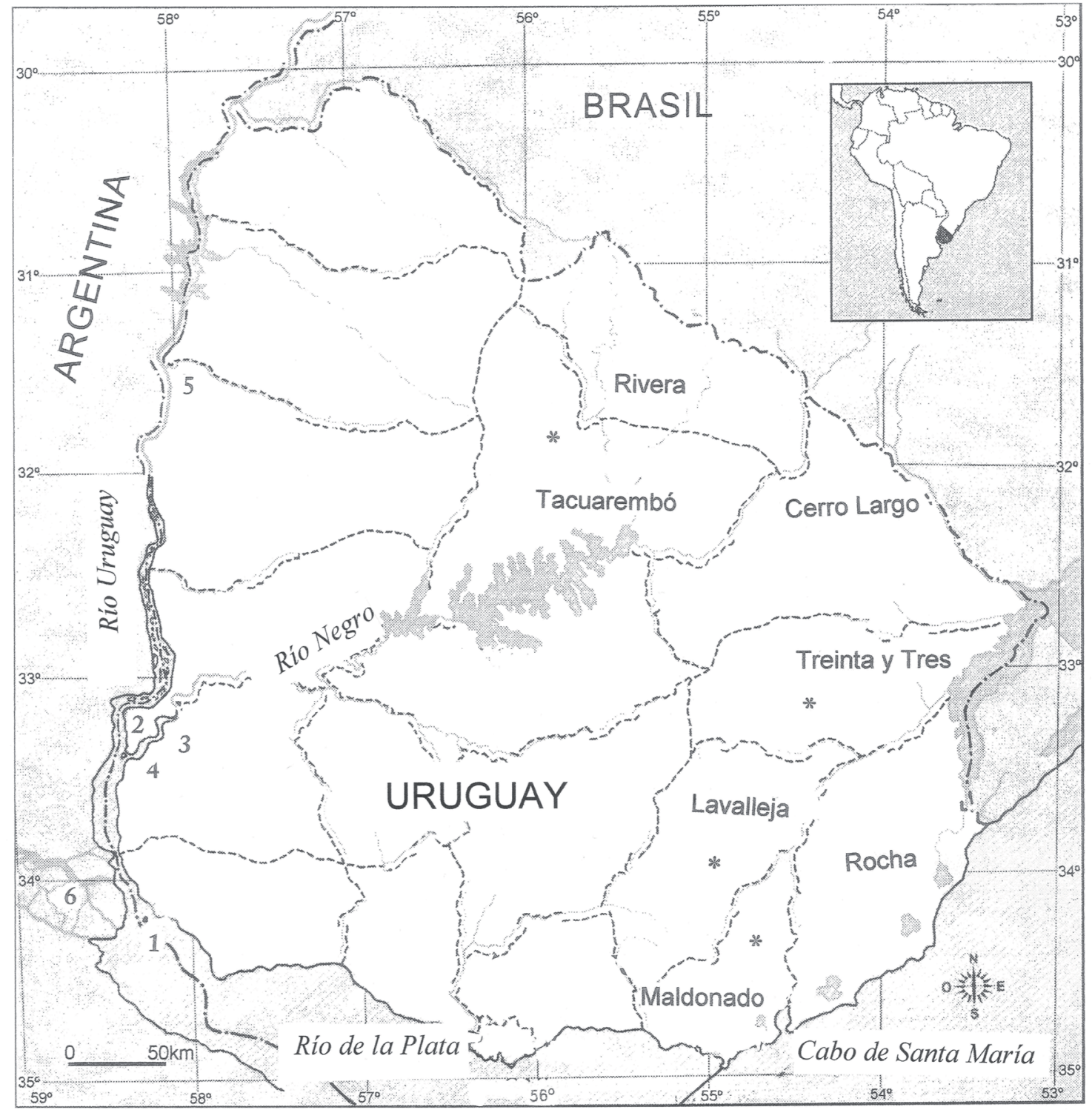

Fig. 2. Lugares mencionados por Bonpland en el manuscrito $\mathrm{N}^{\mathrm{o}} 2044$ y la existencia real de Ilex paraguariensis silvestre en Uruguay. Se reportan árboles autóctonos de "yerba mate" en los departamentos Cerro Largo, Lavalleja, Maldonado, Rivera, Rocha, Tacuarembó y Treinta y Tres. Referencias: 1) Isla Martín García (Argentina), 2) Rincón de las Gallinas, 3) los arroyos Maulas, 4) Villa de Soriano, 5) Hervidero, 6) delta del Paraná-Uruguay (Argentina). Los lugares de colección de Ilex paraguariensis visitados por el autor se muestran con un asterisco (*).

bien puede haberse tratado de una situación similar a la de varios árboles frutales exóticos que se encontraron en el delta del Paraná, como fue sugerido por d'Orbigny (Orbigny, 1945a). Su posible existencia en algunas islas de la región a principios del siglo XIX parece haber sido casual, probablemente en calidad de plantas adventicias, relacionados con los viajes efectuados a lo largo de estos ríos, en lugar de ser el producto de intentos de cultivo por los jesuitas. Martínez Crovetto (1995) opinó que la presencia de I. paraguariensis en el delta argentino era posible, pero que con el correr del tiempo esos árboles habrían desaparecido.

En otro orden de cosas, los comentarios de Bonpland sobre la existencia de Ilex paraguariensis en varios lugares de Uruguay, parecen aún más extraños. 
Algunos de los lugares mencionados en el manuscrito son relativamente fáciles de identificar casi dos siglos más tarde-" "Rincón de las Gallinas" o "Rincón de Haedo", y asimismo el "Hervidero", "Soriano", "Maulas" (Fig. 2)-, mientras que otros son datos desconcertantes. Por ejemplo, la "Sierra de Santa María cercana al Hervidero" no aparece en ningún mapa moderno ni en obras geográficas antiguas que haya podido consultar.

La exacta localización geográfica de un lugar llamado "Cerritos" supuestamente cercano a la actual ciudad de Soriano (departamento Soriano, República Oriental del Uruguay) es confusa: los "cerritos de indios" son sitios arqueológicos indígenas relativamente comunes en los departamentos uruguayos Tacuarembó y Rocha, pero son menos abundantes en el departamento Soriano. Es interesante recordar que también en el dpto. Soriano, según Tomé (1952) el Dr. Juan de La Peña, en su quinta de Mercedes hacia 1840, habría poseído plantaciones de yerba mate.

El autor del presente estudio cree que la mayoría de los datos mencionados por Bonpland para Uruguay en el documento $\mathrm{N}^{\circ}$ 2044, que pueden haber sido escritos entre 1818 y 1824, reflejan la circunstancial ignorancia del naturalista de la geografíay ambiente uruguayos, ya que Bonpland aún no había podido viajar por ese país en esa etapa de su vida. Años más tarde, el botánico francés se familiarizó con el territorio uruguayo, especialmente con el rio Uruguay, conocimientos que pudo adquirir a lo largo de numerosos viajes (1832-1855) que realizó por el territorio de ese país y/o por el rio Uruguay (Bell, 2010). En consecuencia, me parece factible que este informe de circa 1820 sobre la "Sierra de Santa María" pueda en realidad referirse mejor a la situación de las serranías bajas del actual departamento Maldonado, que se encuentran relativamente cerca del Cabo Santa María (también conocido como Cabo Rocha) sobre la costa atlántica uruguaya (lugar que quizás es más conocido por su cercanía a la localidad La Paloma-Isabelle, 1835-, si bien algunos viajeros muy antiguos lo han confundido con lo que actualmente es la localidad Punta del Este-Arredondo, 1958-).

En estas serranías bajas del departamento Maldonado, Ilex paraguariensis realmente existe silvestre (ver Material Examinado).

El caso del "Rincón de las Gallinas", una zona fácilmente reconocible en la actualidad, también llamada "Rincón de Haedo" es bastante curioso. Esta región, dentro de lo que se reconoce como vegetación riparia del río Uruguay, es desde antiguo una zona explotada forestalmente y de cría de ganado. No obstante, sus tipos de vegetación difieren bastante de los que están característicamente relacionados con especies de Ilex (véase "Ilex paraguariensis y sus requerimientos ambientales"). A pesar de esta antigua afirmación de Bonpland sobre la presencia de Ilex paraguariensis en esta región de Uruguay (Bonpland, 1818-1824), el mismo naturalista viajó en 1832 por la zona y aunque se refirió al "Rincón de las Gallinas" y realizó comentarios sobre la vegetación observada a lo largo de su viaje por el rio Uruguay, ya no mencionó la presencia de la yerba mate en la región (Bonpland, 1832). Adicionalmente, ninguno de los viajeros naturalistas contemporáneos reportaron su existencia allí (ni A. de Saint Hilaire, que viajó por esos lugares en 1820-1821 -Jenkins, 1946-, ni A. Isabelle, quien realizó un viaje por la región en 1833, registraron datos sobre I. paraguariensis allí -Arechavaleta, 1906; Isabelle, 1835; Saint Hilaire, 1823 "1822"-). Si bien por supuesto no se trata de acabados estudios ambientales (Gautreau, 2006) debe reconocerse el valor testimonial de los relatos de todos estos viajeros. Otros viajeros científicos contemporáneos en el Uruguay como F. Sellow y A. d'Orbigny, tampoco reportaron la ocurrencia de I. paraguariensis en este país (Arechavaleta, 1905; Herter, 1947 -“1945”-; Orbigny, 1945a, 1945b; Weiss, 1940).

Aunque hoy en día bastante modificada, la vegetación ribereña del río Uruguay en tales latitudes no incluye la "yerba mate" entre una lista de varios representantes nativos de la vegetación del "Alto Uruguay" o Paranaense que habita estos lugares (Brussa Santander \& Grela González, 2007).

\section{Ilex paraguariensis y sus requerimientos ambientales}

Con relación a los requerimientos ambientales que posibilitan la existencia 
de Ilex paraguariensis en condiciones naturales, los de índole climática parecen ser determinantes. Ellos implican precipitaciones anuales no inferiores a los $1200 \mathrm{~mm}$ y una distribución uniforme de las mismas a lo largo del año. De otra forma, la existencia de esta planta en regiones de menor precipitación pluvial anual solamente se puede verificar si el suministro de agua proviene de otras fuentes tales como manantiales o los cursos de agua disponibles (Fig. 3). Otras variables climáticas que son importantes según la distribución actual del género Ilex en el planeta, tales como las restricciones térmicas (Loizeau \& al. 2005), parecen importar menos para el caso de I. paraguariensis. El promedio anual de precipitaciones en el área de distribución natural de esta especie en Sudamérica varía entre los 1300 y 2200 mm. Además, esta zona está aproximadamente comprendida entre el área determinada por las isotermas anuales de $15.5^{\circ}$ y $25.5^{\circ} \mathrm{C}$ (Aranda, s.f.). De acuerdo con la clasificación climática de Köppen (1948), esta área exhibe predominantemente climas tipo Cfb (templado-cálidos, sin estación seca, veranos frescos), junto con áreas climáticas $\mathrm{Cfa}$ (templado-cálidos, sin estación seca, veranos cálidos) y seguidas por áreas con climas tipo Cwa (templado-cálidas, inviernos secos) y, en zonas muy reducidas, con presencia de climas Aw (o de savanas tropicales) -Malheiros de Oliveira \& Rotta 1985-. Uruguay posee un clima tipo Cfa (Brussa Santander \& Grela González, 2007). Los tipos de vegetación presentes en la extensa región sudamericana que disfruta de estos climas (SE del Brasil, oriente paraguayo y nordeste argentino) pertenecen biogeográficamente a la Provincia

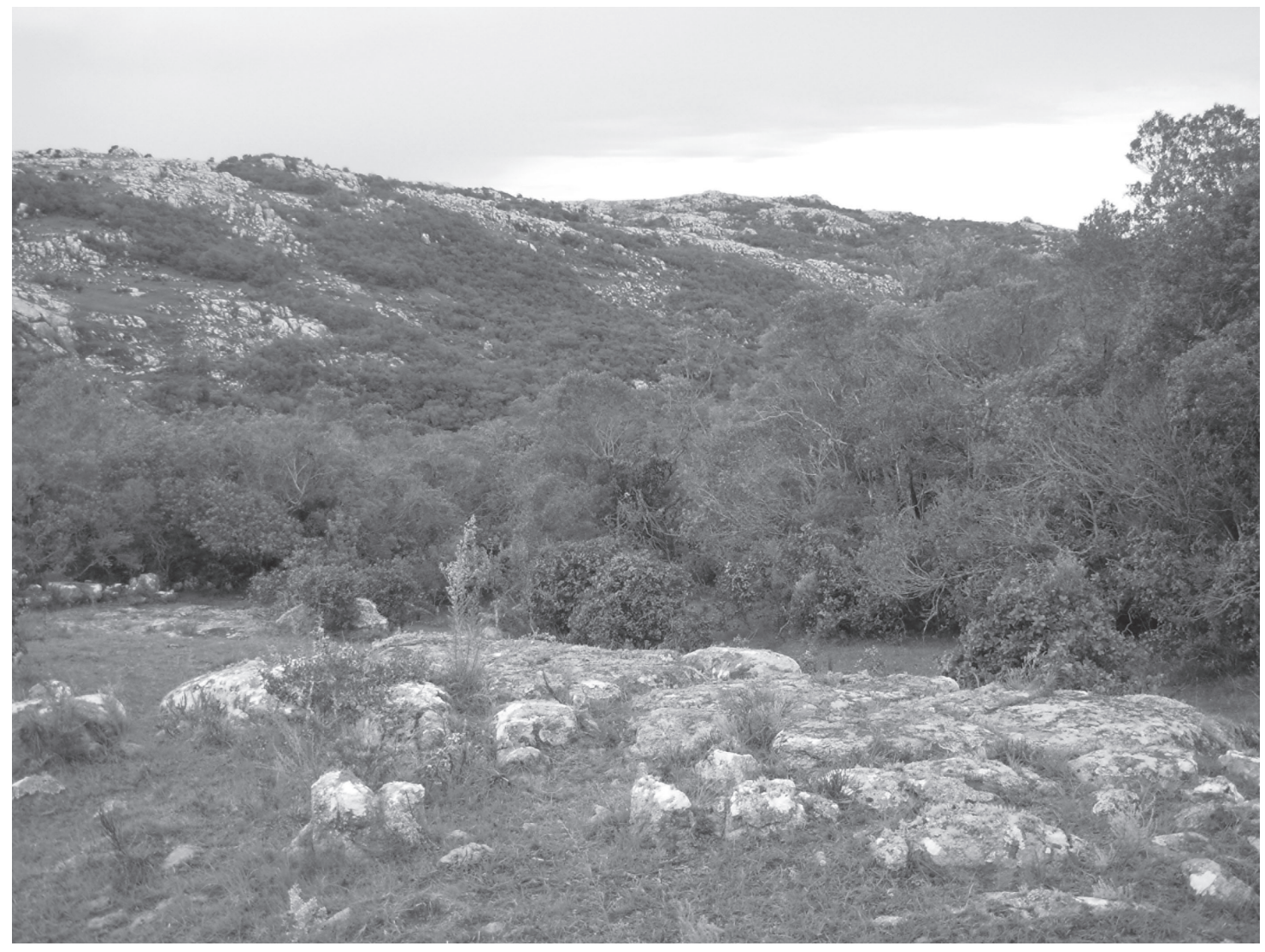

Fig. 3. Fisonomía de la vegetación en el enclave más austral -conocido- para Ilex paraguariensis silvestre en Uruguay: el fondo húmedo de un valle serrano en las "Asperezas" del Departamento Maldonado, las comunidades de vegetación dominantes que circundan el valle no son arbóreas (foto del autor, 2009). 
Paranaense del Dominio Amazónico, en tanto que Uruguay se incluye en su totalidad en la Provincia Pampeana del mismo dominio (Cabrera \& Willink, 1980). Aunque para Brasil (Occhioni \& Hatschbach, 1972) y para Argentina (Martínez-Crovetto, 1981) fueran publicadas algunas nóminas -no exhaustivas- de las especies que cohabitan con I. paraguariensis, y algunas de estas entidades incluyen taxones que existen en Uruguay, la distribución de muchas de esas especies no alcanza a llegar a este último país, como es el caso de Araucaria angustifolia (Bertol.) O. Kuntze, característico representante de la comunidad vegetal donde crece silvestre la yerba mate.

\section{Selección de material silvestre uruguayo de Ilex paraguariensis:}

Maldonado: Ruta 109, aproximadamente 10 $\mathrm{km}$ al sur de Aiguá, Estancias "Don Lisandro Yerbal”, 34 $16^{\prime} \mathrm{S} *$, $54^{\circ} 37^{\prime} \mathrm{W}, \pm 330$ m s.n.m, 2-IV-2009, G. C. Giberti et al. 699, 700 (BAF), 701 (BAF).- Arroyo Yerbal, 26-I-1923, J. Schroeder (Herbarium C. Osten) 19690 (BA, BAF).

Lavalleja: Aproximadamente a $8 \mathrm{~km}$ del mojón del km 197 de la ruta 8, Estancia "La Lorencita", $33^{\circ} 55^{\prime} \mathrm{S}, 54^{\circ} 41^{\prime} \mathrm{W}, \pm 190$ m s.n.m., 3-IV-2009, G. C. Giberti et al. 703 (BAF).- Estancia "La Lorencita", 8-I-1923, C. Osten 19689 (BAF).

Tacuarembó: Aproximadamente $10 \mathrm{~km}$ al NO de la ciudad de Tacuarembó, Cuchilla de Haedo, Gruta de los Helechos, $31^{\circ} 38^{\prime} \mathrm{S}, 56^{\circ} 02^{\prime} \mathrm{W}, 30$-III2009, G. C. Giberti et al. 691, 692 (BAF).- Sierra Gaona, 1924, C. Osten 17305 (BAF).

Treinta y Tres: cercanías de la Quebrada de los Cuervos, aproximadamente $31 \mathrm{~km}$ al $\mathrm{N}$ de la ciudad de Treinta y Tres, en cañada húmeda del arroyo Cajoncito, $32^{\circ} 56^{\prime} \mathrm{S}, 54^{\circ} 28^{\prime} \mathrm{W}, 188 \mathrm{~m}$ s.n.m., 1-IV-2009, G. C. Giberti et al. 697 (BAF), 698 (BAF).

\section{Conclusiones}

En resumen, en estos viejos manuscritos de Bonpland (Bonpland, 1818-1824), algunos datos sobre la supuesta existencia de Ilex paraguariensis en varios lugares de la República Oriental del Uruguay deberían considerarse erróneos, quizás originados por el desconocimiento circunstancial de este naturalista sobre la geografía y características ambientales de ese país, durante aquellos tempranos tiempos de su residencia en la región de la Cuenca del Plata. Esta situación fue un obstáculo al que fácilmente Bonpland se sobrepuso algunos años después, al efectuar numerosos viajes a través de la cuenca del Uruguay y el territorio oriental (1832-1855); y fue así como esos datos desconcertantes sobre la "yerba mate" no se repitieron en sus manuscritos posteriores. La existencia confirmada de Ilex paraguariensis nativa en Uruguay se ve respaldada por exsiccata coleccionada en ambientes silvestres de los departamentos Cerro Largo, Lavalleja, Maldonado, Rocha, Tacuarembó, Treinta y Tres -y también posiblemente en Rivera (Brussa Santander \& Grela González, 2007)de la República Oriental del Uruguay (Alonso Paz, 2008; Arechavaleta, 1900; Brussa Santander \& Grela González, 2007; Giberti, 1995, 2008; Grela \& Brussa, 2003; Grondona, 1954; Herter, 1931 “1930”; Lombardo, 1964). Por otra parte, existen algunas referencias a antiguos cultivos de" yerba mate" efectuados en diversos departamentos de Uruguay (Berro, 1914; Tomé, 1952), pero estos datos no deben confundirse con otros que indican la presencia real del árbol de "yerba mate" entre la vegetación nativa.

El mismo Bonpland un año más tarde de su primer hallazgo de "yerba mate" de diciembre de 1818 viajó por el Delta de los ríos Uruguay y Paraná sin poder encontrar más árboles de "yerba" -salvo cuando revisitó la isla Martín García (1819)-.

\section{Agradecimientos}

Este estudio fue posible gracias al CONICET (Argentina). Debo expresar también mi profundo reconocimiento a

* Nota. Esta es la latitud más austral de Ilex que se haya confirmado para América del Sur, mientras que para todo el planeta, solamente llex mitis Radlk. (de Sudáfrica) llega más al sur. 
fitotecnistas de la Estación Experimental Cerro Azul del INTA (Argentina), a varios científicos del INIA (Uruguay), a colegas independientes y productores agropecuarios uruguayos, quienes tuvieron la amabilidad de aconsejarme o realizar viajes conmigo por el país hermano. Este artículo está dedicado a la memoria del Ing. Agr. Raúl N. Martínez Crovetto, destacado taxónomo, conocedor de la flora misionera y precursor de estudios etnobotánicos en Argentina, entusiasta bibliófilo que asimismo recopiló gran cantidad de informaciones sobre la "yerba mate".

\section{Bibliografía}

ALONSO PAZ, E. 2008. Yerba mate: aspectos botánicos y biogeográficos. Exposición durante el Primer Simposio Internacional sobre Yerba Mate y Salud, Montevideo, 17 - 18 de octubre 2008.

ARANDA, D. s.f. Área de Distribución Natural de la Yerba Mate. Miscelánea 14, Estación Experimental Agropecuaria Misiones, INTA. 17 pp.

ARECHAVALETA, J. 1900. Flora Uruguaya. Enumeración y descripción breve de las plantas..., Tomo 1. Establecimiento Tipo-Litográfico Oriental, Montevideo. XXI + 493 pp.

—. 1905. Fl. Urug. ..., Tomo 2. Talleres A. Barreiro y Ramos, Montevideo. XXVII + 375 pp.

—. 1906. Fl. Urug. ..., Tomo 3. Talleres Gráf. A Barreiro y Ramos, Montevideo. 504 pp.

ARREDONDO, H. 1958. Viajeros visitantes del Uruguay. Revista Sociedad "Amigos de la Arqueología" 17: 7-313.

BELL, S. 2010. A Life in Shadow. Aimé Bonpland in Southern South America, 1817 - 1858. Stanford Univ. Press, Stanford, California. XIII + 320 pp.

BERRO, M. B. 1914. La Agricultura Colonial. Imprenta Artística de J. J. Dornaleche, Montevideo. $4+351$ pp.

BONPLAND, A. 1818-1824a. Hierba del Paraguay o mate. Manuscrito 2044, Archivo Bonpland, Facultad de Farmacia y Bioquímica, Universidad de Buenos Aires.

. 1818-1824b. Voyage dans le Guazú fait sur la sumaca de Dn. Francisco Belgrano. Août 1819. Manuscrito 2044, Archivo Bonpland, Facultad de Farmacia y Bioquímica, Universidad de Buenos Aires.

1832. Journal Voyage de Buenos Ayres à Sn. Borja par 1' Uruguay. Manuscrito 1698, Archivo Bonpland, Facultad de Farmacia y Bioquímica, Universidad de Buenos Aires.

1867. Notas sobre la utilidad de trabajar los yerbales, empleando un procedimiento distinto del que se emplea hasta hoy en día por los rutineros que los benefician. Revista Farm. 5: 270-276.

- 1949. Notas sobre yerbales. Traducción por el Doctor Juan Pujol. Nota preliminar por el Doctor Fernando A. Coni Bazán. Lilloa 18: 361-371.

BROWN, N. E. 1892. Notes on certain plants Yielding Paraguay Tea. Bull. Misc. Inform. 65-66: 133-137.

BRUSSA SANTANDER, C. A. \& I. A. GRELA GONZÁLEZ. 2007. Flora Arbórea del Uruguay. Con énfasis en las especies de Rivera y Tacuarembó. COFUSA, Montevideo. 543 pp.

BURKART, A. \& N. M. BACIGALUPO (eds.). 2005. Flora Ilustrada de Entre Ríos (Argentina). Parte 4. Dicotiledóneas Arquiclamídeas. B: Geraniales a Umbelliflorales. Colección Científica del INTA VI, Buenos Aires. VIII + 627 pp.

CABRERA, A. L. (ed.). 1965. Flora de la Provincia de Buenos Aires. Parte $4^{\mathrm{a}}$. Oxalidáceas a Umbelíferas. Colección Científica del INTA IV, Buenos Aires. I $+418 \mathrm{pp}$.

— \& A. WILLINK. 1980. Biogeografía de América Latina. Segunda edición. Monografía 13. Serie de Biología. OEA, Washington, DC: 122 pp.

CASTELlanOS, A. 1963. Bonpland en los países del Plata. Revista Acad. Colomb. Ci. Exact. 12: 57-86.

DELESSERT, F. 1854. Communication faite par M. François Delessert d'une lettre de M. de Bonpland. Compt. Rend. Hebd. séances Acad. Sci. 38: 434438.

DOMÍNGUEZ, J. A. 1924. Archives inédites de Aimé Bonpland. II, Journal de Botanique. Trab. Mus. Farmacol. Fac. Ci. Méd. Buenos Aires 42.

GALARZA, J. B. 1914. La Yerba - Mate. Bol. Minist. Agric. (Buenos Aires) 18: 44-101.

GAUTREAU, P. 2006. Relatos de crisis ambiental en el Rio de La Plata. Una evaluación geográfica de 300 años de relatos de "destrucción" de los bosques uruguayos (siglos XVIII al XIX). Tesis (Traducción H. Inda). Université des Sciences et Technologie de Lille, $357 \mathrm{pp}$.

GIBERTI, G. C. 1979. Las especies argentinas del género Ilex L. (Aquifoliaceae). Darwiniana 22: 217-240.

-1988. Aquifoliaceae paraguayas: notas críticas en el género Ilex. En Spichiger, R. (ed.), Notulae ad Floram paraquaiensem 14-17, Candollea 43: 417-420.

1990. Bonpland's manuscript name for the yerba mate and Ilex theezans C. Martius ex Reisseck (Aquifoliaceae). Taxon 39: 663-665.

—. 1994 Aquifoliaceae. En Hunziker, A. T. (ed.), Flora Fanerogámica de Argentina Fascículo 1 (157), Museo Botánico, IMBIV, Córdoba, Argentina.

. 1995. Aspectos oscuros de la corología de Ilex paraguariensis St. Hil. En Winge, H., A. G. Ferreira, J. E. de A. Mariath \& L. C. Tarasconi (eds.), ErvaMate: biologia e cultura no Cone Sul, pp. 289-300. 
Edit. da Universidade, UFRGS, Porto Alegre. 2008. Aquifoliaceae. En Zuloaga, F. O., O. Morrone \& M. J. Belgrano (eds.), Catálogo de las Plantas Vasculares del Cono Sur (Argentina, Sur de Brasil, Chile, Paraguay y Uruguay. Vol. 2 Dicotyledoneae (Acanthaceae - Fabaceae (Abarema - Schizolobium), pp. 1143-1146. Monogr. Syst. Bot. Missouri Bot. Gard. 107.

GRELA, I \& C. BRUSSA. 2003. Relevamiento florístico y análisis comparativo de comunidades arbóreas de Sierra de Rios (Cerro Largo- Uruguay). Agrociencia 7: 11-26.

GRONDONA, E. M. 1954. Historia de la yerba mate. II. Revista Argent. Agron. 21: 9-24.

HAMY, T. J. E. 1906. Aimé Bonpland médicin et naturaliste explorateur de l'Amérique du Sud, sa vie, sa correspondence... . Librarie Orientale \& Américaine, E. Guilmoto, Paris. XCVII + 300 pp.

HERTER, W. 1931 “1930“. Florula Uruguayensis plantae vasculares II. Enumeratio plantarum... . Sumptibus rei publicae, Montevideo. 191 pp.

—. 1947 "1945“. Auf den Spuren des Naturforscher Sellow und Saint - Hilaire. Bot. Jahrb. Syst. 74: 119-149 + mapa.

HOSSARD, N. 2001. Aimé Bonpland (1773-1858) médecin, naturaliste explorateur en Amérique du Sur. A l'ombre des arbres. L'Harmattan, Paris. $1+$ $237 \mathrm{pp}$.

ISABELLE, A. 1835. Voyage a Buénos - Ayres et a Porto Alègre, par la Banda - Oriental, les Missions d'Uruguay et la province de Rio-Grande-do-Sul (de 1830 à 1834)... . Imprimerie de J. Morlent, Place de la Comédie, Havre. 618 pp. + 1 mapa.

JENKINS, A. E. 1946. Introductory essay. Chronica bot. 10: 5-21.

KÖPPEN, W. 1948. Climatología. Fondo de Cultura Económica, México - Buenos Aires. 478 pp.

KRAPOVICKAS, A. 2008. Bonpland, sesquicentenario de su muerte. Bonplandia (Corrientes) 17: 5-11.

LOESENER, T. 1901. Monographia Aquifoliacearum. I. Nova Acta Acad. Caes. Leop. - Carol. German. Nat. Cur. 78.

LOIZEAU, P.- A., G. BARRIERA, J.- F. MANEN, \& O. BROENNIMANN. 2005. Towards an understanding of the distribution of Ilex L. (Aquifoliaceae) on a World-wide scale. Biologiske Skrifter. 55: 501-520.

LOMBARDO, A. 1964. Flora arbórea y arborescente del Uruguay. Con clave para determinar las especies ( $2^{\text {da }}$ ed.). Taller. Gráficos Bouzot, Montevideo. 151 pp.

LOURTEIG, A. 1977. Amado Bonpland. Bonplandia (Corrientes) 3: 269-317.

MALHEIROS DE OLIVEIRA, Y.M. \& E. ROTTA.
1985. Area de distribuição natural de Erva Mate (Ilex paraguariensis St.Hil.). Documentos EMBRAPA 15: 17-35.

MARTÍNEZ-CROVETTO, R. 1981. Composición florística de yerbales naturales de los alrededores de San Pedro (Misiones). Publicación Técnica 3. Facultad de Ciencias Agrarias, Universidad Nacional del Nordeste: 1-11 + 3 láminas.

. 1995. La Yerba Mate. Maravilla de América. Museo de Motivos Argentinos "José Hernández", Buenos Aires. 223 pp.

MIERS, J. 1861. On the history of the maté Plant and the different species of Ilex employed in the preparation of the "Yerba Maté" or Paraguay Tea. Ann. Mag. Nat. Hist. $3^{\text {rd. }}$ ser., 8: 389-401.

MUELLO, A. C. 1946. Yerba Mate. Su Cultivo y explotación. Enciclopedia Agropecuaria Argentina. 31. Editorial Sudamericana, Buenos Aires. 217 pp.

MÜNTER, J. 1883. Ueber Mate (Maté) und die Mate - Pflanzen Süd - Amerika's. Mitth. Naturwiss. Vereine Neu - Vorpommern 14: 103-24 + 2 tabl.

NEUMANN, K. 1858. Bemerkungen A. Bonpland's über die Verbreitungssphäre des Paraguay - Thee's. Z. Allg. Erdk. 5: 76-83.

OCCHIONI, P. \& G. HATSCHBACH. 1972. A vegetação arbórea dos ervais do Paraná. I. Leandra. Año 2 (3): $1-59$.

ORBIGNY, A. D. D'. 1945a. Viaje a la América meridional ... .Vol. I. Editorial Futuro, Buenos Aires. 7 - 439 pp.

—. 1945b. Viaje a la América meridional ... .Vol. III. Editorial Futuro, Buenos Aires. 835 - 1232 pp.

QUESADA, V. G. 1857. La Provincia de Corrientes. Imprenta del Orden, Buenos Ayres. 5-113 pp. + errata.

SAINT HILAIRE, A. DE. 1823 “1822”. Aperçu d'un voyage dans l'intérieur du Brésil, la province Cisplatine et les Missions dites du Paraguay. Mém. Mus. Hist. Nat. 9: 307-380.

SPEGAZZINI, C. 1905. Flora de la Provincia de Buenos Aires. Anales del Ministerio de Agricultura. Sección de Biología Vegetal. Imprenta de M. B. Biedma é Hijo, Buenos Aires. XIV + 161 pp.

TOMÉ, E. 1952. La Yerba Mate. Almanaque Banco de Seguros del Estado, Montevideo. 85-88 pp.

WEISS, C. S. 1940. Sôbre a extremidade meridional da cordillera do Brasil, na Província de S. Pedro do Sul e na Banda Oriental ou Estado de Monte Video; conforme às coleções do Sr. Fr. Sellow, por Weiss. Tradução do Gal. Bertholdo Klinger. Boletim do Centro Rio-Grandense de Estudos Históricos 2: 35$98+5$ láminas.

Original recibido el 18 de julio de 2011; aceptado el 24 de noviembre de 2011. 